\title{
The IMF control of the Martian bow shock and plasma flow in the magnetosheath. Predictions of 3-D simulations and observations
}

\author{
E. Dubinin ${ }^{1,2,5}$, K. Sauer ${ }^{1,5}$, M. Delva ${ }^{3,5}$, and T. Tanaka ${ }^{4}$ \\ ${ }^{1}$ Max-Planck-Institut für Aeronomie, D-37191 Katlenburg-Lindau, Germany \\ ${ }^{2}$ Space Research Institute, Russian Academy of Sciences, 117810 Moscow, Russia \\ ${ }^{3}$ Space Research Institute, Inffeldgasse, 12, 8010, Graz, Austria \\ ${ }^{4}$ Communications Research Laboratory, Koganei-shi, Tokyo, Japan \\ ${ }^{5}$ International Space Science Institute (ISSI), Hallerstrasse 6, 3012 Bern, Switzerland
}

(Received August 4, 1997; Revised June 26, 1998; Accepted July 8, 1998)

\begin{abstract}
Recent three-dimensional simulations of the Martian plasma and magnetic field environment found some unique effects - a strong asymmetry of the bow shock and the magnetic field draping, asymmetry of plasma inflow to the wake, formation of secondary shocks near the boundary of the magnetospheric cavity. The predictions of the models are testified using the Phobos-2 data. It is shown, that, although, the models reproduce some features of the bow shock and plasma flow around the planet, the real picture appears to be more variable and complicated. We suppose that addition of planetary plasma originated from the atmosphere/exosphere and effects of mass-loading is necessary modification of three-dimensional models.
\end{abstract}

\section{Introduction}

Planetary bodies without intrinsic magnetic field, but with substantial ionospheres/atmospheres possess magnetospheres formed by draping of the passing solar wind flux tubes around the obstacle. Mass-loading due to pickup of newly created planetary ions add mass to the flow, slowing it and leading to an additional draping of the magnetic field. Venus gave us the first example of a planet with a draping magnetic field configuration controlled by the orientation of the interplanetary magnetic field (IMF). The magnetic field orientation introduces an asymmetry into the plasma-field distribution. The equator to pole asymmetry of shock distances, which can be as large as $10 \%$, is a consequence of the varying speed of propagation of fast magnetosound waves (Cloutier, 1976; Romanov, 1978; Russell et al., 1988). Another asymmetry appears because the gyroradius of heavy ions at Venus is comparable to the planetary radius. This so-called asymmetry of mass-loading is controlled by the direction of the motional electric field. As a result, the field draping in the magnetosheath is weaker in the hemisphere, in which pickup ions starting to move along their cycloid trajectories may encounter the planet's obstacle, and stronger where they move outward without encounters (Phillips et al., 1987). This effect of finite gyroradius of picked up ions is probably also responsible for the difference in ionopause altitude (Phillips et al., 1988). Alexander et al. (1986) have observed the venusian bow shock at larger distances in the hemisphere where the motional electric field is pointed outward the magnetosphere. East-west draping asymmetry in the tail, which is controlled by the IMF sector polarity, and a north-south asymmetry related with a direction of the mo-

Copy right (c) The Society of Geomagnetism and Earth, Planetary and Space Sciences (SGEPSS); The Seismological Society of Japan; The Volcanological Society of Japan; The Geodetic Society of Japan; The Japanese Society for Planetary Sciences. tional electric field have been reported by McComas et al. (1986). Slavin et al. (1989) have found an asymmetry in the thickness of the tail boundary and suggested that the draped field in the magnetic tail may be mainly supplied over the hemisphere with the locally electric field outward to the equator plane.

Mars, like Venus, has no detected intrinsic magnetic field, but possesses an extended exosphere and atmosphere. Therefore, one may expect to observe also essential asymmetry in the magnetic field and plasma flow around the planet. Analyzing the IMF control of the position of the Martian bow shock Zhang et al. (1991) have found a median $0.21 R_{\mathrm{M}}$ difference, the shock being at the larger distance in the northern hemisphere where the picked up ions go upward. Pole-to equator asymmetry with larger distance in the polar direction, was also noted. However, Mars, as compared with Venus, has the unique feature. Mars is only half the radius of Venus and its bow shock may be not efficient at thermalizing the solar wind protons because of lack of room. Brecht (1997a, b) has suggested that the traditional description, based on MHD approach, is not valid for Mars, and the bow shock position might be mainly controlled by kinetic effects. Using a fully three-dimensional hybrid particle code, Brecht (1997a, b) has investigated a magnetic topology of Mars. One of the fascinating features is a large asymmetry of the bow shock in the north-south plane, with a large flaring in the direction, which is opposite to the solar wind motional electric field. One of the purposes of the paper is to test asymmetry of the Martian bow shock using the magnetic field measurements onboard the Phobos-2 spacecraft.

Another approach, based on the 3-D MHD modeling, was also applied to simulate the solar wind interaction with Mars, and could be tested on the base of the Phobos-2 data. Using a 3-D MHD code, Tanaka (1993) have found the asymmetry 


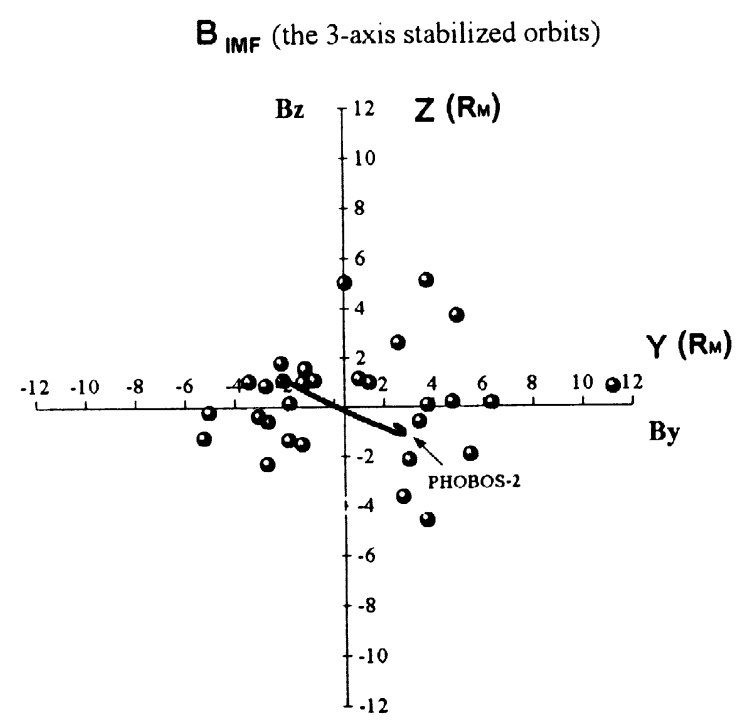

(a)

\section{BOW SHOCK POSITION IN VB FRAME}

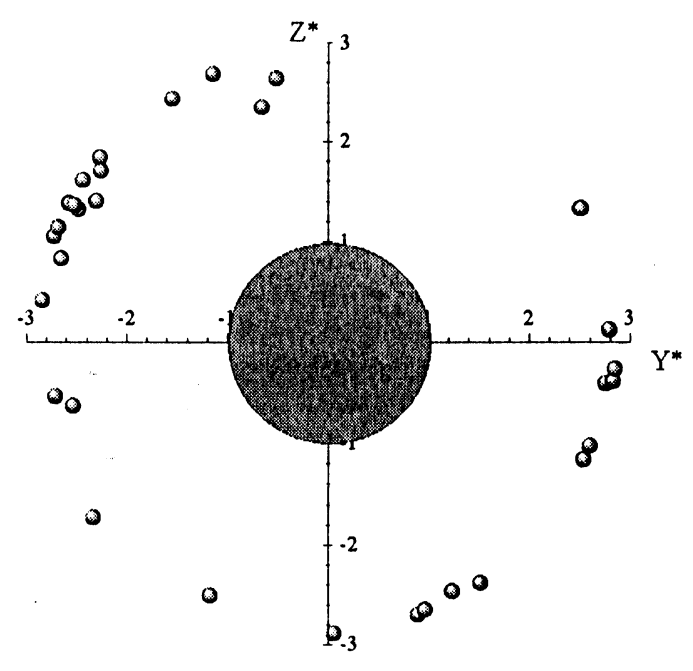

(b)

Fig. 1. (a) Cross flow component of the IMF for the set of three-axis stabilized orbits. The solid line gives the $Y Z$-projection of the spacecraft trajectory in Mars Solar Orbital (MSO) coordinates. (b) Positions of the BS crossings in the $Y^{*} Z^{*}$ plane.

of ion flow into wake in equatorial and polar regions due to a difference of the action of $\boldsymbol{j} \times \boldsymbol{B}$ and plasma pressure forces. Because of the $\boldsymbol{j} \times \boldsymbol{B}$ forces, flow is accelerated in the polar region and decelerated in the equatorial plane, providing asymmetry of the bulk velocity in polar and equatorial magnetosheath. As an effect of plasma reacceleration, secondary shocks appear at the nightside near the terminator while draped flux tubes sink into the wake.

\section{Asymmetry of the Bow Shock. Predictions and Observations}

Most observations near Mars were done when Phobos 2 was settled on the circular orbit $\left(R \sim 2.8 R_{\mathrm{M}}\right)$. The bow shock (BS) was crossed usually near the terminator. For the study of the solar wind interaction with planets, like Venus and Mars, having draped magnetospheric configurations, the magnetic $\boldsymbol{V} \boldsymbol{B}$ reference frame is more sensible one (Dolginov et al., 1981; Saunders and Russell, 1986). This frame has the $X$-axis, which points to the Sun and the cross-flow magnetic field component along the new $Y^{*}$-axis. The motional electric field $(-1 / c \boldsymbol{V} \times \boldsymbol{B})$ is always pointed to the $+Z^{*}$ direction. The average cross-flow magnetic field was determined and then, was used to rotate the vector of the spacecraft position into the $X Y^{*} Z^{*}$-reference frame. Large $B_{Z}$-component in the solar wind can "rotate" the spacecraft trajectory into the polar region of the $\boldsymbol{V B}$ system. Therefore, since the IMF varies, the spacecraft orbits in the $\boldsymbol{V B}$ frame may cover the entire $Y^{*} Z^{*}$ plane (Dubinin et al., 1996a).

In some circular orbits the spacecraft was three axis stabilized, while in another orbits Phobos- 2 was in a spinning mode with the axis of rotation intended to be the instrumental $X$-axis of the spacecraft and always pointing to the Sun. However, the axis of rotation was really deviating from the $X$-axis of the spacecraft and only the approximate direction of the axis of rotation was known. Additional despun processing of the data was done (Delva et al., 1994). In this study we use separately both sets of the data.

Figure 1(a) gives the cross flow component of the IMF for the set of three-axis stabilized orbits when a probable error of the despinning procedure is excluded. Since the IMF may vary, orbits were divided in two parts: inbound and outbound, and the average cross-flow magnetic field vectors were calculated for those passes independently. The solid line shows the $Y Z$-projection of the spacecraft trajectory in Mars Solar Orbital (MSO) coordinates with the $X$-axis toward the Sun, the $Y$-axis pointing in opposite direction of the planet's orbital velocity and the $Z$-axis perpendicular to $X$ and $Y$. In most cases, the vector of the IMF was near the ecliptic plane. However, on several orbits the IMF rotated into a north-south orientation, giving rise to polar crossings of the BS in the $(\boldsymbol{V B})$ reference frame. Figure 1(b) shows the $Y^{*}$ - and $Z^{*}$-coordinates of the BS crossings. It is seen that the orbits sample not only magnetic equatorial plane, but also mid and high latitudes. The shape of the bow shock is not dramatically asymmetric in the $Y^{*} Z^{*}$ plane, although variations in the $X$-position of the crossings lead to a more scattering while projecting onto the $X-R$ plane $\left(R=\left(Y^{* 2}+Z^{* 2}\right)^{1 / 2}\right)$ (Fig. 2(b)).

Figure 2(a) presents the $X$ - and $\theta$-coordinates of the BS positions, where $\theta$ is the angle between the $Y^{*}$-axis and the projection of the radius-vector of the bow shock onto the $Y^{*} Z^{*}$ plane. Angles $90^{\circ}$ and $270^{\circ}$ give the direction to the north and south. Circles, crosses and squares depict the BS position for the polar $\left(45^{\circ}-135^{\circ}\right.$ and $\left.225^{\circ}-315^{\circ}\right)$ and equatorial orbits respectively. Larger $X$-values correspond to larger flaring angles (Fig. 2(b)), because the subsolar location of the bow shock is rather stable (Schwingenschuh et al., 1990). If the effects of finite proton gyroradius are crucial for the BS asymmetry, as was suggested by Brecht (1997a, b), one could expect that $X_{\mathrm{S}}>X_{\mathrm{N}}, X_{\mathrm{E}}$, where $X_{\mathrm{S}}, X_{\mathrm{N}}$ and $X_{\mathrm{E}}$ are the $X$-coordinates of the BS in the southern, northern caps and near the magnetic equator respectively. Although statis- 


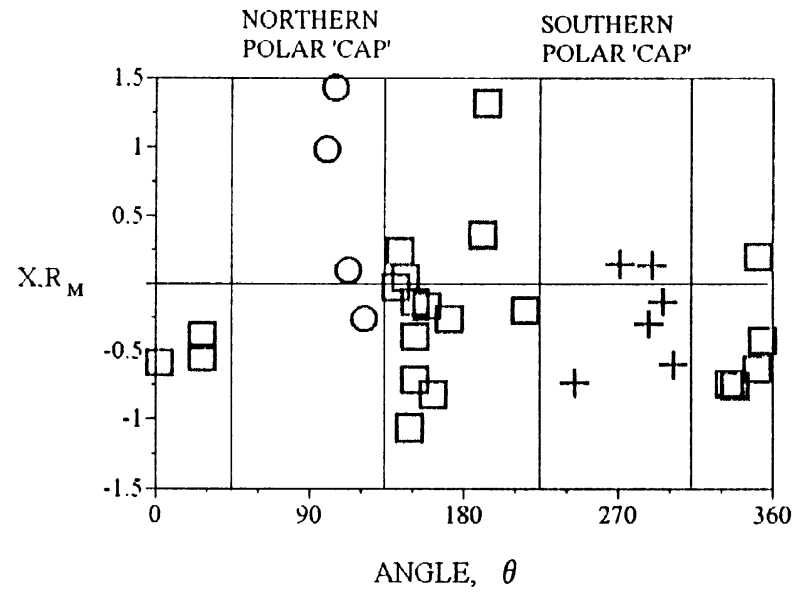

(a)

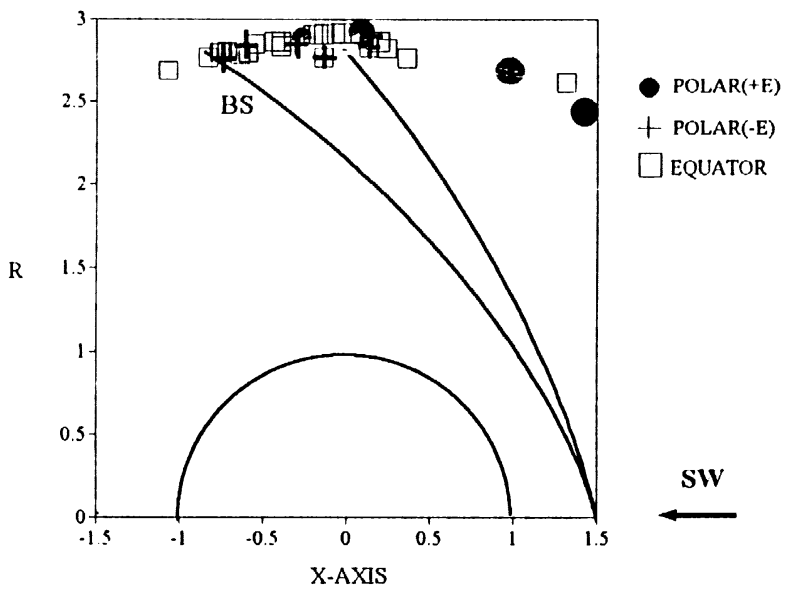

(b)

Fig. 2. (a) Positions of the BS on stabilized orbits in the $X-\theta$ coordinates. Angles $90^{\circ}$ and $270^{\circ}$ give the direction to the north and south. Circles, crosses and squares depict the BS position for the polar $\left(45^{\circ}-135^{\circ}\right.$ and $\left.225^{\circ}-315^{\circ}\right)$ and equatorial orbits respectively. (b) Locations of the BS in the cylindrical reference frame.

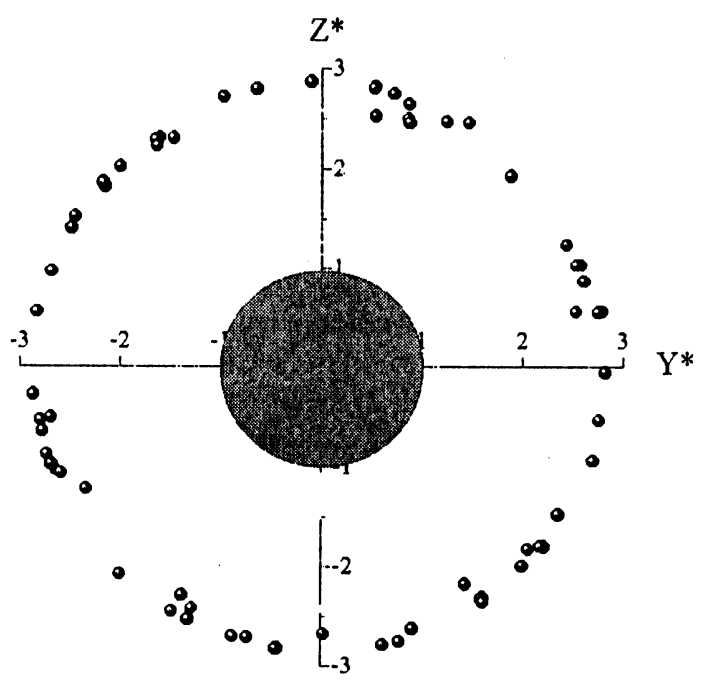

(a)

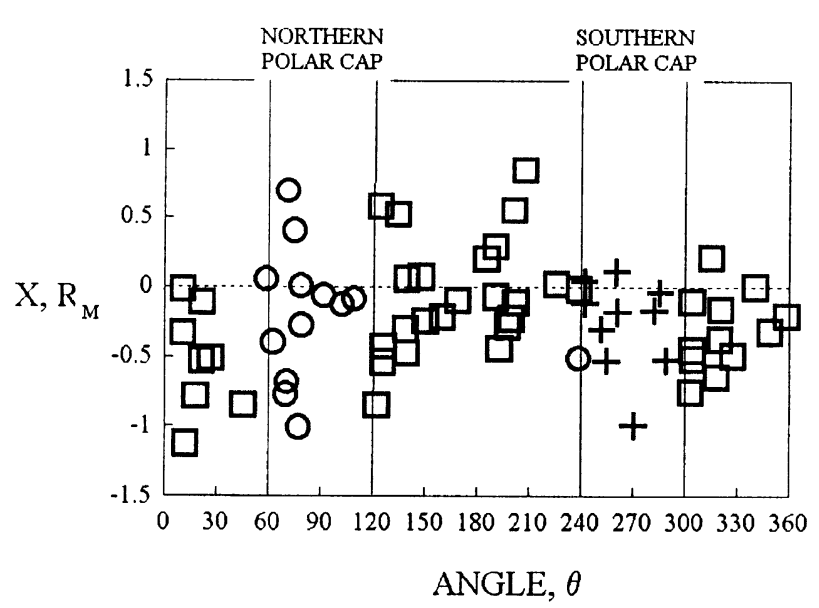

(b)

Fig. 3. (a) Positions of the BS in the $Y^{*} Z^{*}$ plane for the set of spinning orbits. (b) $X-\theta$ coordinates of BS are given.

tics of the BS crossings in the caps is poor such trend is not observed. The median values of $X_{\mathrm{S}}, X_{\mathrm{N}}$ and $X_{\mathrm{E}}(-0.22,0.54$ and $-0.33 R_{\mathrm{M}}$ ) indicate to an opposite trend, although two upstream excursions of the $\mathrm{BS}\left(X \geq 1 R_{\mathrm{M}}\right)$ were associated with low values of $M_{\mathrm{A}}$.

Analysis of the data obtained while the spacecraft was spinning, brings better statistics and similar results. Figure 3(a) shows positions of the BS in $Y^{*} Z^{*}$ plane. A uniform coverage of all latitudes is evident. It is worth noting that position of the BS is unambiguously identified from the distinct shock ramp for most of the orbits. In case of any uncertainty, the magnetic field data were complemented by the plasma measurements. "Indentations" at the BS are distributed rather randomly. Figure 3(b) gives the $X$ - and $\theta$-coordinates of the BS. As in the previous case of three- axis stabilized orbits, the most evident aspect is a significant variability of the BS. Mean $X$-values for crossings in the southern and northern polar caps are $X_{\mathrm{S}}=-0.28 \pm 0.3 R_{\mathrm{M}}$ and $X_{\mathrm{N}}=-0.22 \pm 0.47 R_{\mathrm{M}}$. A variability of the BS locations is well manifested by that error bars are bigger than mean values.

Realizing that statistical approach may smear out and loose expected effects because of interference of different controlling factors, we classified the data by considering a probable influence of several parameters to the BS location. A value of the motional electric field $(-1 / c \boldsymbol{V} \times \boldsymbol{B})$ may control an obstacle asymmetry. A value of the solar wind flux $\left(n V_{\mathrm{sw}}\right)$ influences to charge-exchange and electron impact processes of pickup ion production. Mach number is also important, at least, while at its small values (Slavin et al., 1993). Solar 
wind ram pressure may contribute to a shape and position of the obstacle. Figure 4 presents dependence of the $X_{\mathrm{bs}}$ on these parameters. Weak dependence of the terminator bow shock on the ram pressure is evident. It is worth noting that Verigin et al. (1993) also found that the BS position was practically independent on the solar wind pressure. The flaring angle decreases $\left(X \sim-0.5 R_{\mathrm{M}}\right)$ and fluctuations of the BS location begin to fail with increase of the solar wind flux. Similar trend to a less variability of the BS is also observed with increase of the motional electric field. However, for "normal" solar wind conditions a wide spread of data-points remains.

To identify another factors which may influence to the shape of the bow shock we excluded the observations made for extreme conditions: $n V_{\mathrm{sw}}>1.5 \cdot 10^{8} \mathrm{~cm}^{-2} \mathrm{~s}^{-1}, M_{\mathrm{A}}<4$ and $E>3 \mathrm{mV} / \mathrm{m}$. Figure $5(\mathrm{a})$ depicts the $X$ - and $\theta$ coordinates of the BS for the remainder of the data. An
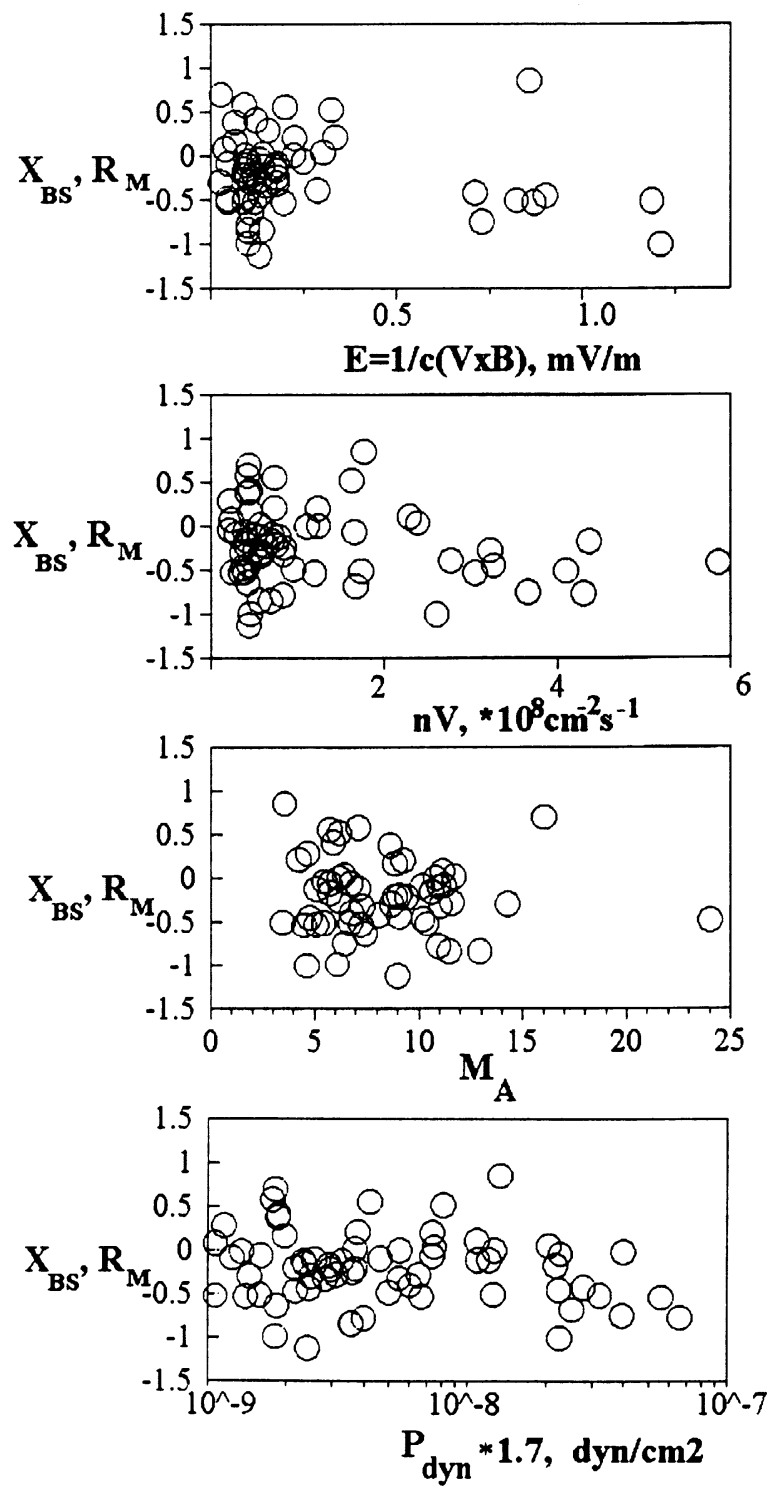

Fig. 4. Dependence of the $X_{\mathrm{BS}}$-coordinate on the motional electric field $(-1 / c \boldsymbol{V} \times \boldsymbol{B})$, the solar wind flux $\left(n V_{\mathrm{sw}}\right)$, Alfven Mach number and the ram pressure.

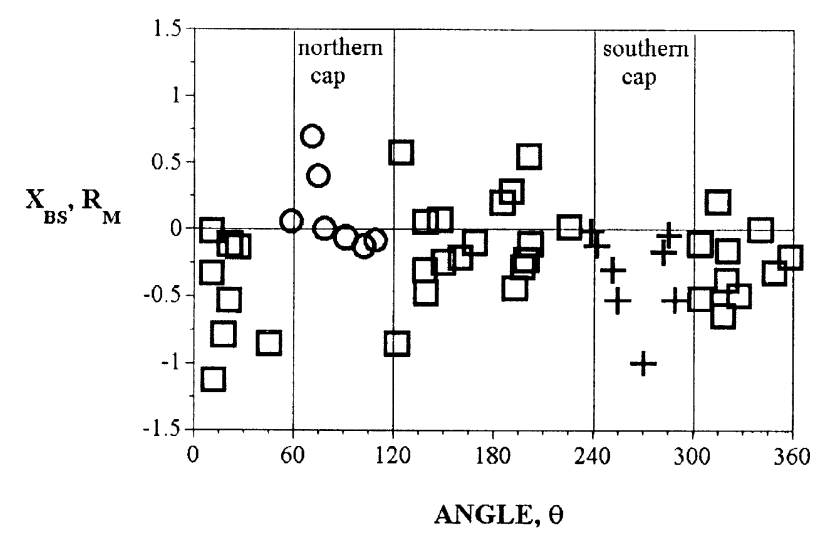

(a)

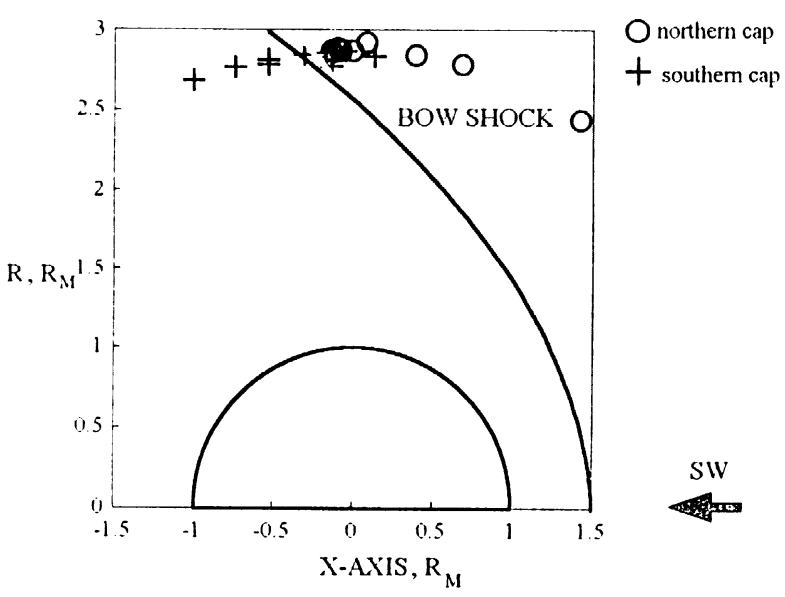

(b)

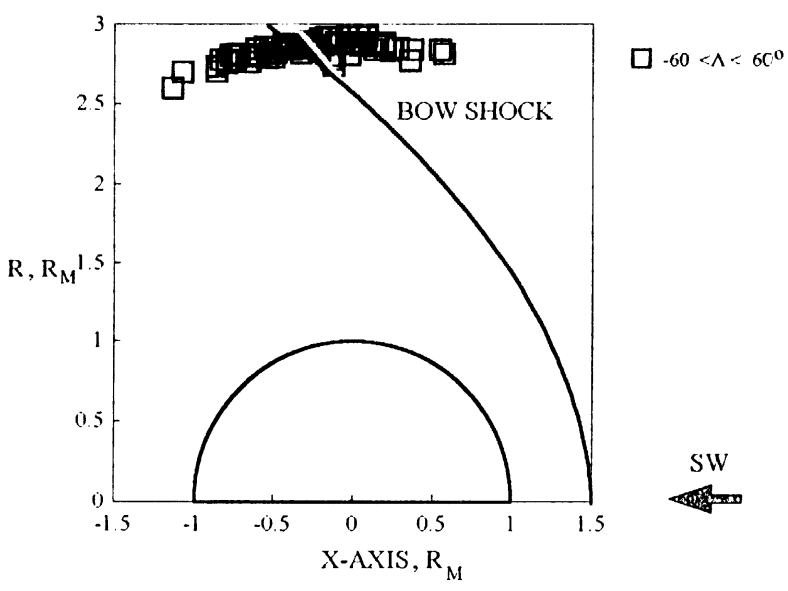

(c)

Fig. 5. (a) The $X-\theta$ coordinates of the BS for "normal" conditions in the solar wind. Effect of north-south asymmetry related with the direction of the electric field appears to be more evident. (b) The $X$ - $R$ coordinates of the BS are given. Larger flaring of the BS is observed in the north hemisphere, in which the motional electric field is pointed out of the equatorial plane. (c) Crossings of the BS reveal large spread of locations at low and middle latitudes. 


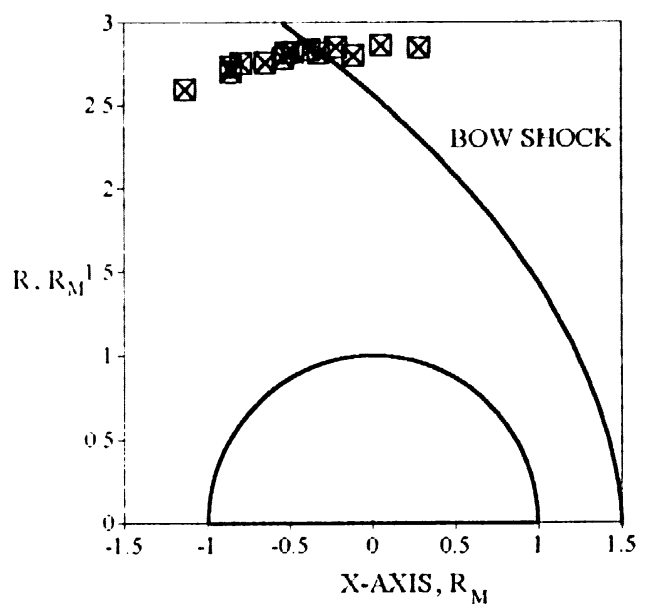

quasiparallel shocks

(a)

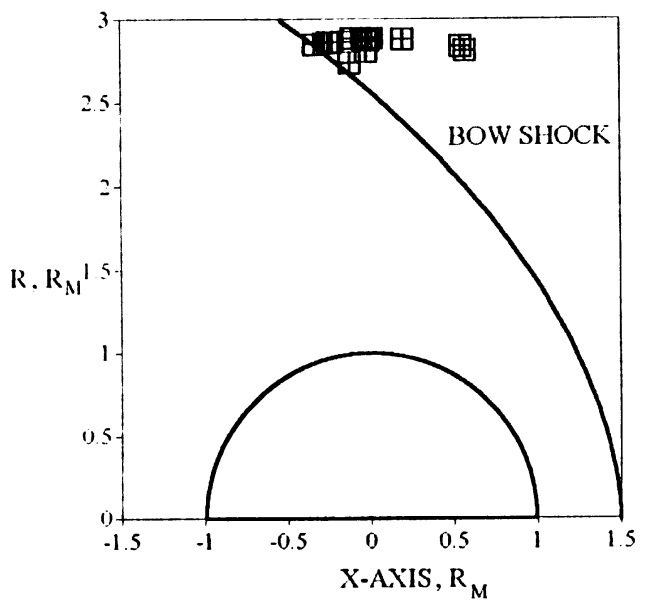

quasiperpendicular shocks

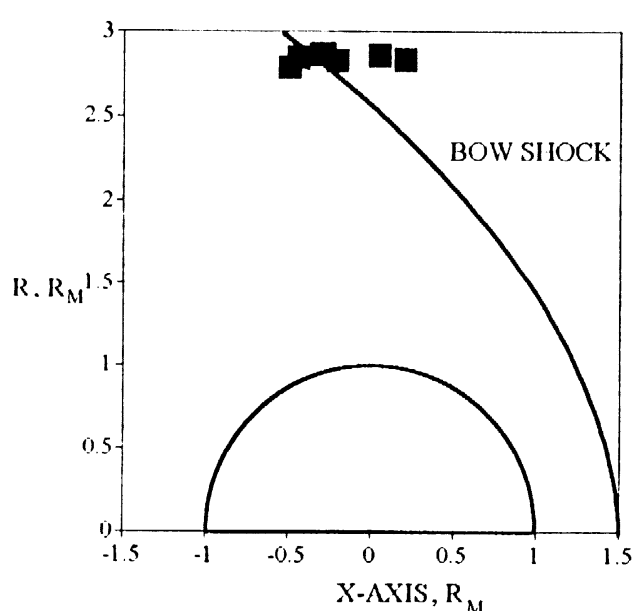

intermediate shocks

(c)

Fig. 6. Locations of the BS at low and middle latitudes classified in dependence on type of bow shock.

effect of north-south asymmetry, related with the direction of the electric field displays more evident. Median values are $X_{\mathrm{S}} \sim-0.24 R_{\mathrm{M}}$ and $X_{\mathrm{N}} \sim 0 R_{\mathrm{M}}$ respectively that corresponds to larger flaring of the BS in the north hemisphere, in which the motional electric field is pointed out of the equatorial plane. Figure 5(b) presents the locations of the BS in cylindrical coordinates. The measurements made during "normal conditions" on the spin stabilized orbits are included for better statistics.

The crossings at low and middle latitudes reveal a large spread of points with excursions of the BS from $X \sim-1 R_{\mathrm{M}}$ to $X \sim+1 R_{\mathrm{M}}$ (Fig. 5(c)). Distribution on the quasiperpendicular (the angle $(\boldsymbol{B n})>45^{\circ}$ ) and quasiparallel (the angle $(\boldsymbol{B n})<45^{\circ}$ ) shocks (n-is local normal to the bow shock) results in a better ordering of the points. Quasiparallel shocks appear to be observed further downstream as compared to the quasiperpendicular shocks (Figs. 6(a) and (b)) with median values of $-0.525 R_{\mathrm{M}}$ and $-0.02 R_{\mathrm{M}}$ respectively. It is worth noting that for the IMF which followed a nominal Parker spiral, quasiperpendicular and quasiparallel shocks were observed on inbound and outbound passes respectively, but the picture was reversed in many cases of anomalous orientation of the IMF. Figure 6(c) gives the locations of the BS for intermediate cases (the angle $\left.\left(\boldsymbol{V}_{\mathrm{sw}} \boldsymbol{B}\right) \sim 90^{\circ}\right)$. Asymmetry between inbound and outbound shocks is small at this IMF configuration. A significant asymmetry between quasiparallel and quasiperpendicular shocks may be caused by different propagation speeds of MHD waves, as well as by "dawndusk" asymmetry of the obstacle. Dubinin et al. (1996a) noticed a remarkable asymmetry of the "obstacle boundary" (see their figure 2). Trotignon et al. (1996) also found a direct relationship between the BS and "obstacle" locations. Both locations vary in the same way and appear to be more closely connected than predicted by gasdynamic models. 
a
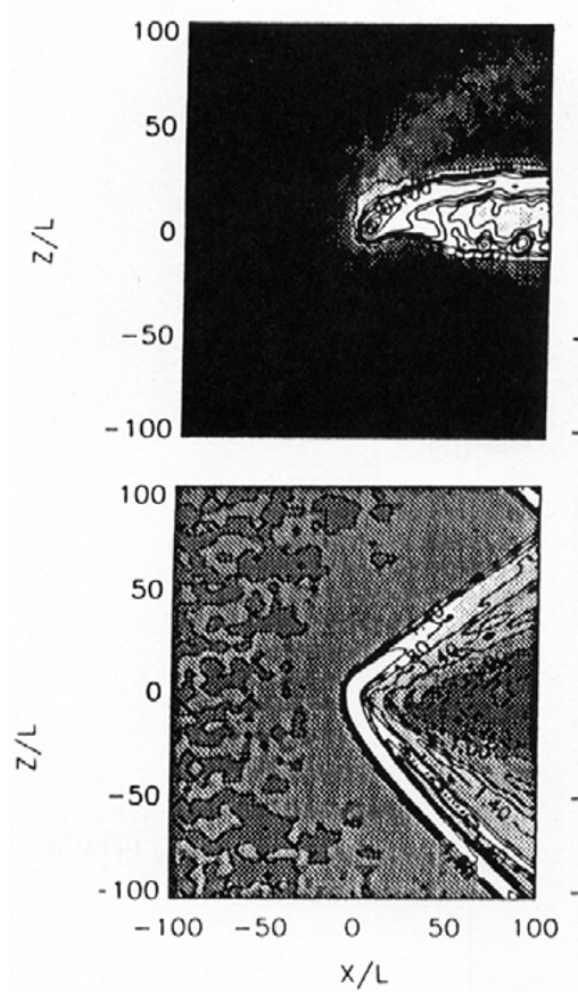

b
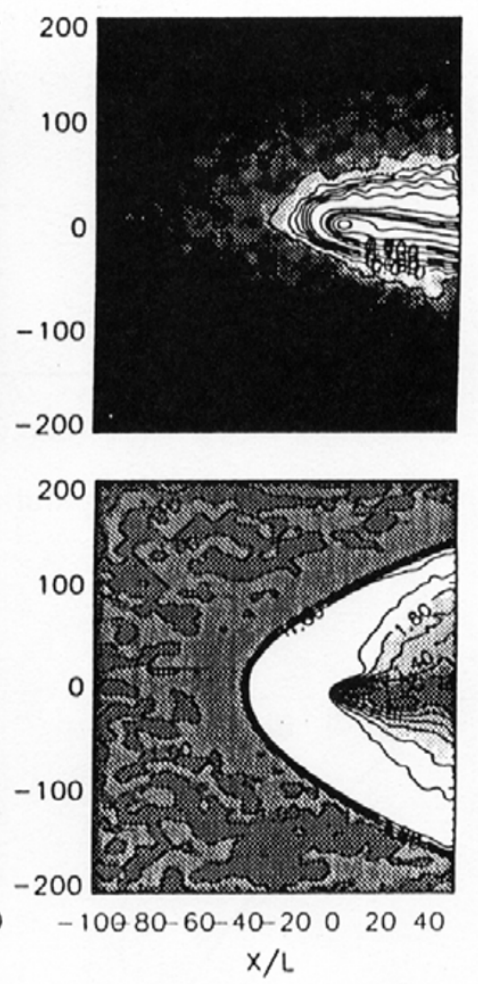

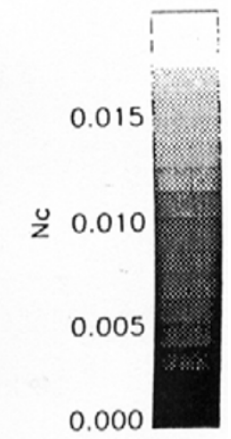

2.0

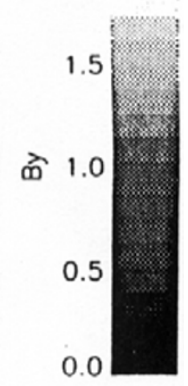

Fig. 7. The distribution of the heavy ion density and the magnetic field component perpendicular to the drawing plane for two different regimes of the solar wind interaction with comet-like obstacles: (a) the gas production rate $Q \sim 7.5 \cdot 10^{25} \mathrm{~s}^{-1}$; (b) $Q>5 \cdot 10^{26} \mathrm{~s}^{-1}$.

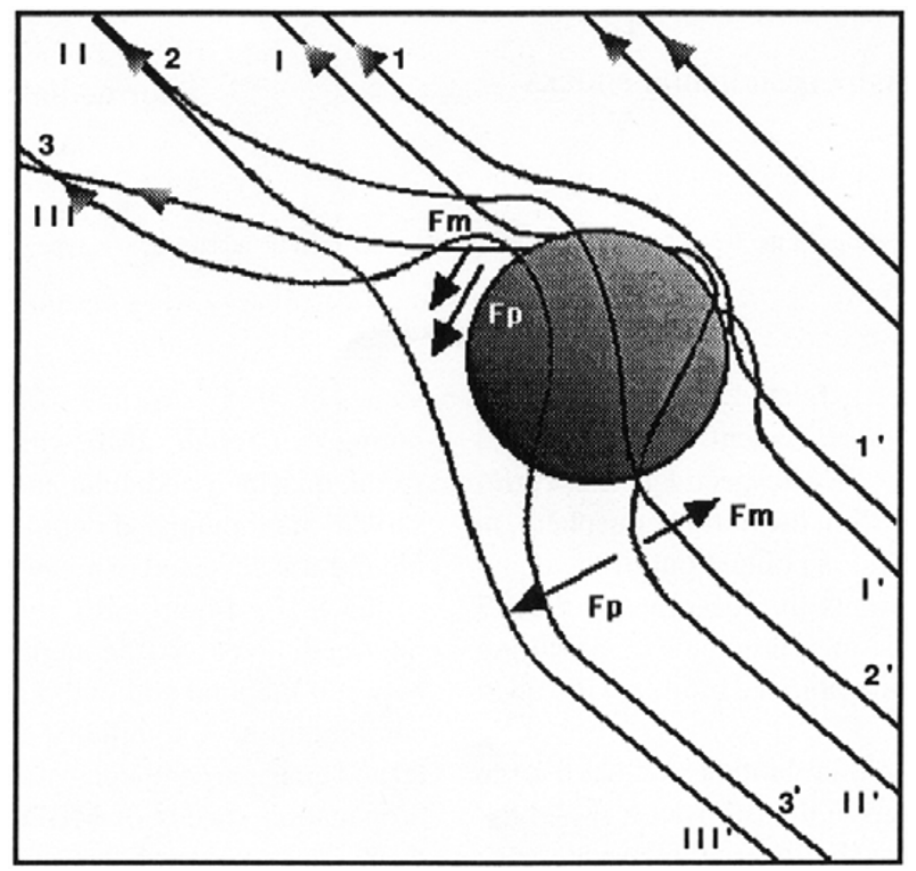

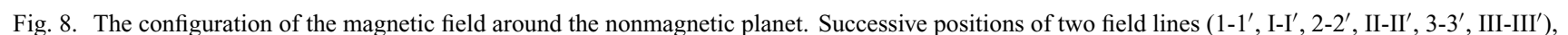
slipping around the upper pole, are shown. The magnetic tension $\left(F_{\mathrm{m}}\right)$ of draped field lines and pressure forces $\left(F_{\mathrm{p}}\right)$ are acting in the same (opposite) directions in the polar (equatorial) regions. 


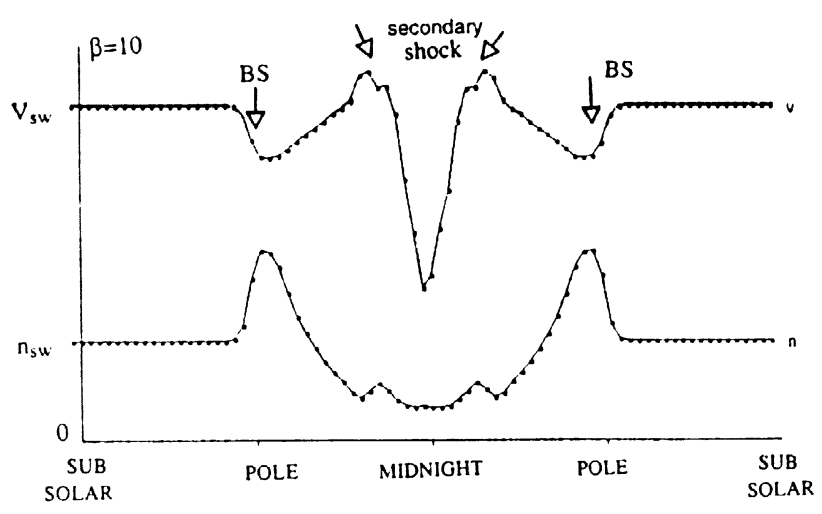

POLAR ORBIT

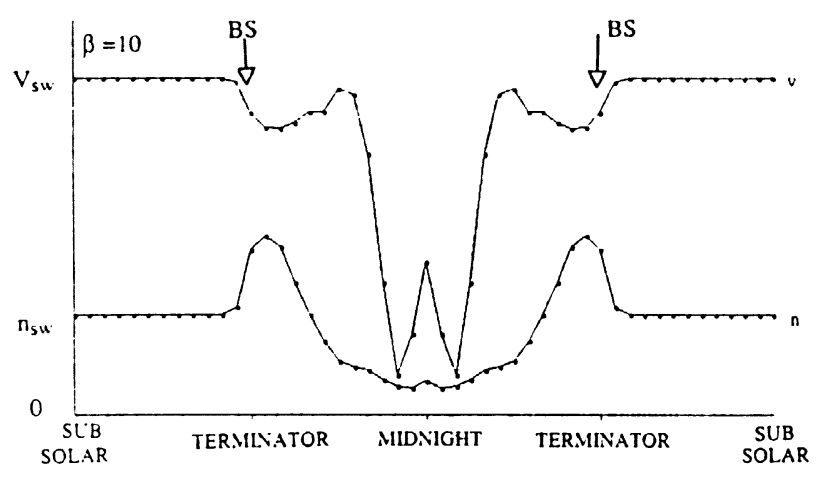

EQUATORIAL ORBIT
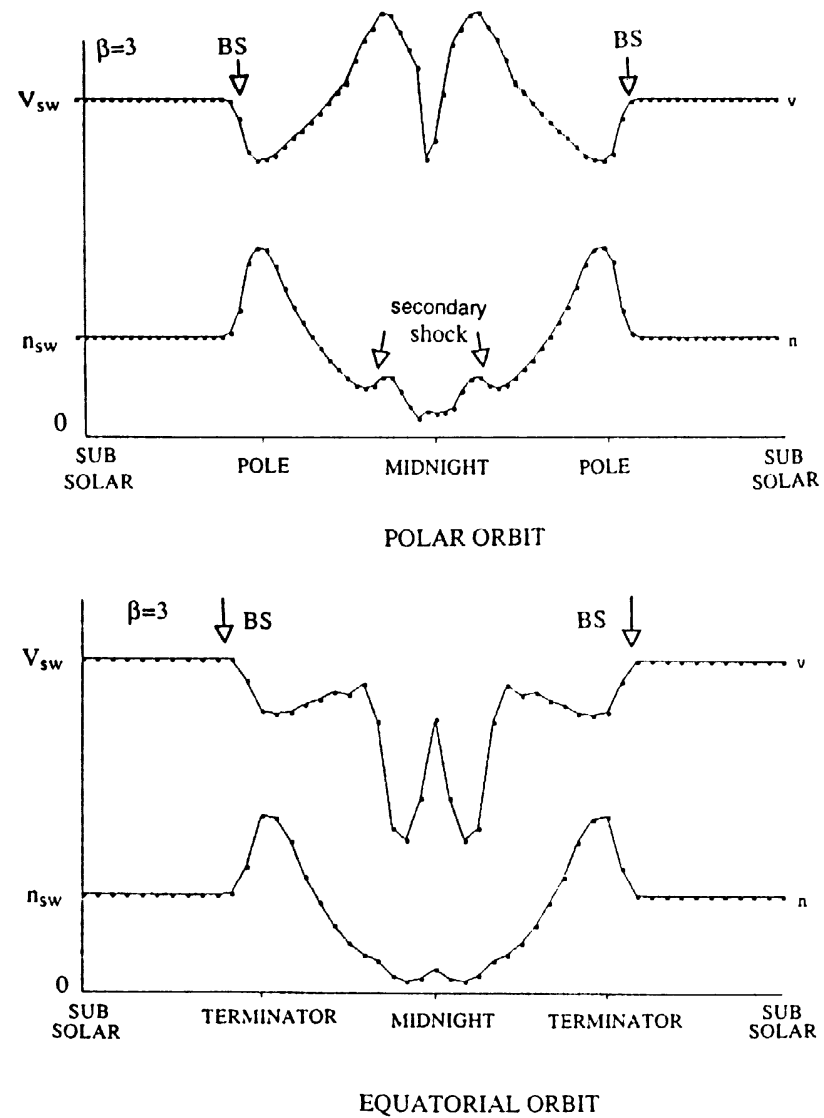

(b)

Fig. 9. Distribution of the plasma number density and the velocity along circular orbits in the equatorial and polar regions $\left(R=2.6 R_{\mathrm{p}}\right)$ in $3 \mathrm{D}-\mathrm{MHD}$ simulations (Tanaka, 1993).

Addressing a question why a shape of the observed bow shock does not contain a "bulge" in the direction opposite to the direction of the motional electric field, which was predicted by the $3 \mathrm{D}$ hybrid simulations, note that planetary ions were not included into the model. As the model obstacle has been taken a conductive sphere. Addition of the planetary heavy ions, favorable moved in the $+Z$ direction, might lead to a decrease of asymmetry associated with a finite proton gyroradius. The 2D bi-ion MHD modeling, which treats the protons and the heavies as separate populations and electrons as a massless fluid shows that the BS occurs at larger distances at the northern half-plane $Z^{*}>0$ (see, for example, figures 1(b)-2(b) from Bogdanov et al., 1996). In the runs by Bogdanov et al. (1996) the magnetic field was perpendicular to the simulation plane, and asymmetry, related with the motional electric field, could be tested. The 2D-bi-ion simulations also demonstrated that the interaction pattern changes with an increase of mass-loading. Recoil effect from heavy ions to the incoming protons results in a significant deflection of the proton flow in the $-Z^{*}$ direction and a modification of a shape of the bow shock with larger flaring in the southern half plane. With further increase of the gas production, the distribution of plasma and the magnetic field appears to be more symmetric. The 2D hybrid code simulations by Lipatov et al. (1997) have confirmed these findings of the 2D-bi-ion MHD modeling. Figure 7, adopted from Lipatov et al. (1997), shows the distribution of the heavy ion density and the magnetic field component perpendicular to the drawing plane for two different regimes of the solar wind interaction with comet-like obstacles. Transition to more symmetric BS with an increase of the comet activity is seen. Larger flaring of the Martian bow shock in the direction of the convection electric field favors regime of "weak" mass-loading near Mars. Although, it is worth noting that the flow pattern around Mars can differ from a comet case because of the existence of the central body-planet with conducting ionosphere as obstacle. Modification of the proton and heavy ion flows will certainly influence to a shape of the bow shock.

Thus, we conclude that many different factors (the value of the motional electric field, the IMF orientation, a shape and features of the obstacle etc.) control the position of the Martian BS. Their combined effect leads to a strong variability of the BS and masks effects predicted by models which are based on simplistic assumptions.

\section{Asymmetry of the Magnetosheath. Predictions and Observations}

The 3D MHD simulations made by Tanaka (1993) have shown an asymmetry of the shocked solar wind flow around nonmagnetized planets. The difference of plasma flows in the polar and equatorial planes (the equatorial plane contains 

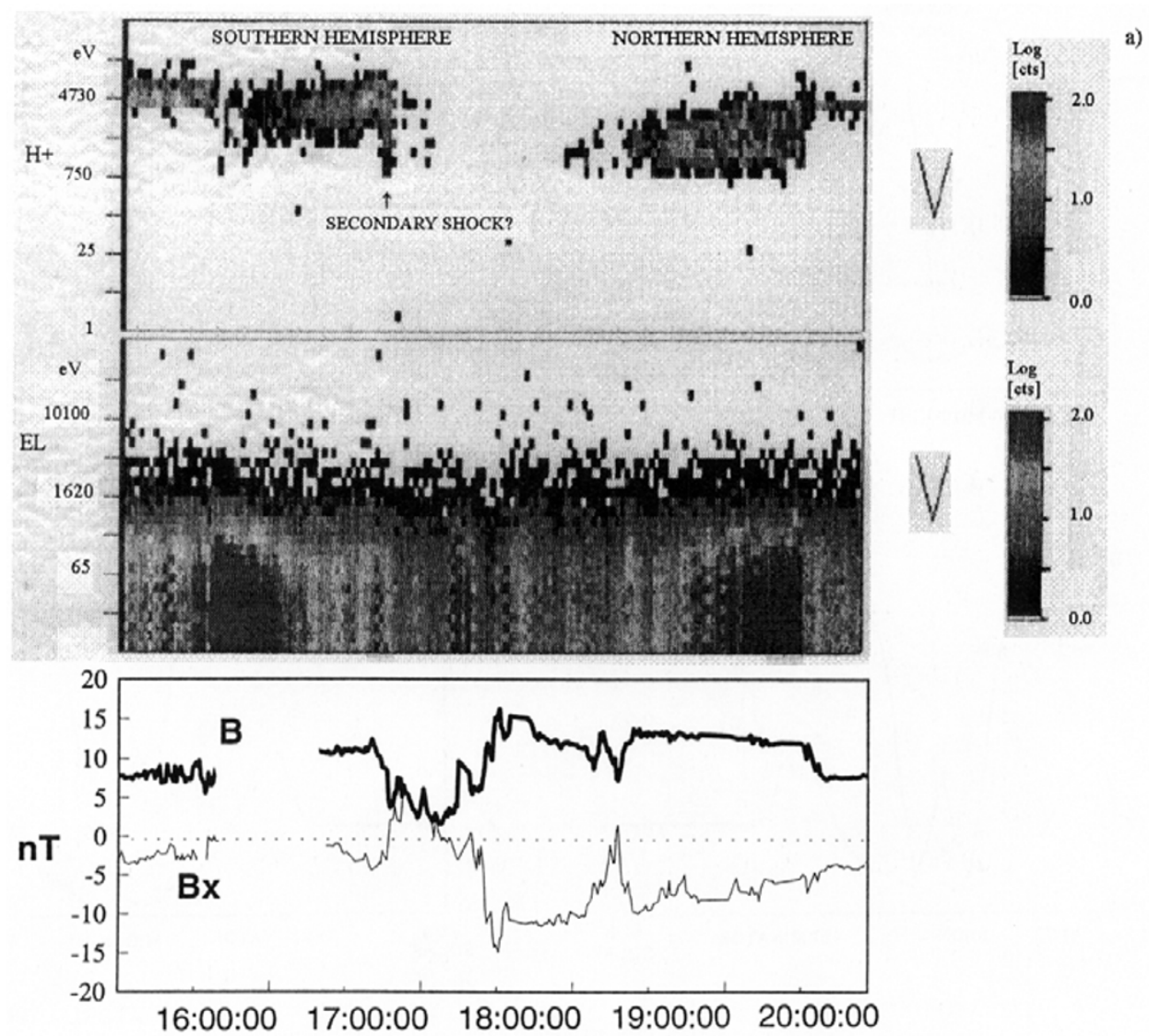

b)

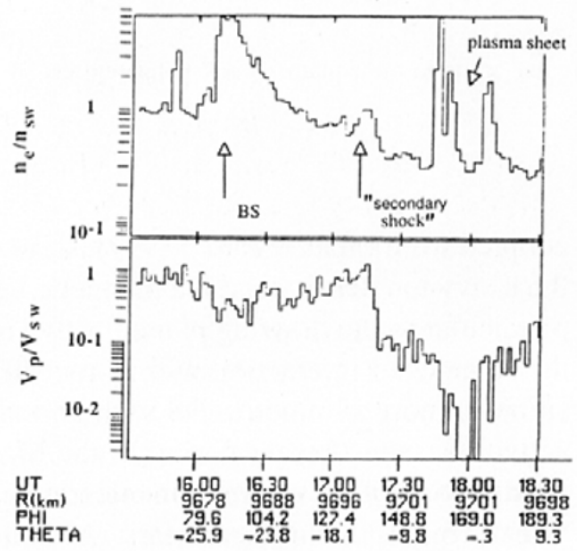

c)

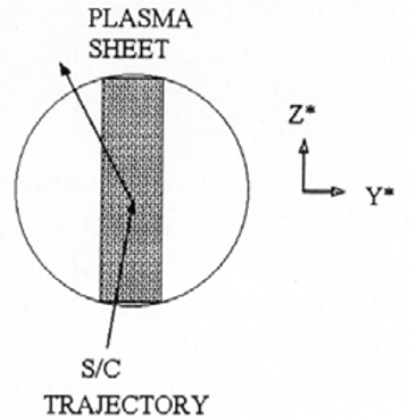

Fig. 10. (a) From top to bottom are energy-time spectrograms of proton and electron fluxes, the $B_{X}$-component and the magnetic field value $B$. (b) Normalized values of fluid parameters (the electron number density, $n_{\mathrm{e}}$ and the proton bulk velocity, $V_{\mathrm{p}}$ ) for the polar orbit on March 13 , 1989. (c) Trajectory of the spacecraft in the $Y^{*} Z^{*}$ plane.

vectors of the IMF and the solar wind speed) is caused by the action of $\boldsymbol{j} \times \boldsymbol{B}$ forces. After passing the stagnation point, the flow is accelerated at flanks. The magnetic tension of the draped field lines accelerates plasma in the polar region and slows it down in the equatorial plane (Fig. 8). As a result, magnetic field lines are slipping around the poles, pushing the shocked plasma. Figure 9 gives the distributions of the simulated number density $n$, and the velocity $V$ along the circular orbits in the equatorial and polar planes at $2.6 R_{\mathrm{p}}$ (planet radii). Under the plasma pressure forces plasma is reaccelerated behind the bow shock. The $\boldsymbol{j} \times \boldsymbol{B}$ force enhances plasma pressure forces near poles and weakens their action in equatorial region. Near the terminator the velocity of reaccelerated plasma, passing through the poles, exceeds the velocity of the undisturbed solar wind. Flow into a wake from the polar side leads to the formation of a secondary shock. Downstream of the BS the plasma pressure decreases because of plasma acceleration. Then, at the secondary shock, plasma density and pressure suddenly increase. Secondary shock was not observed in the equatorial plane, where the action of plasma pressure gradient and magnetic tensions is opposite.

Analyzing the plasma measurements, carried out by the ASPERA instrument, we found only three cases, which indicate to an essential difference in plasma inflow at low and 
PHOBOS-2, ASPERA, 16 MARCH 1989
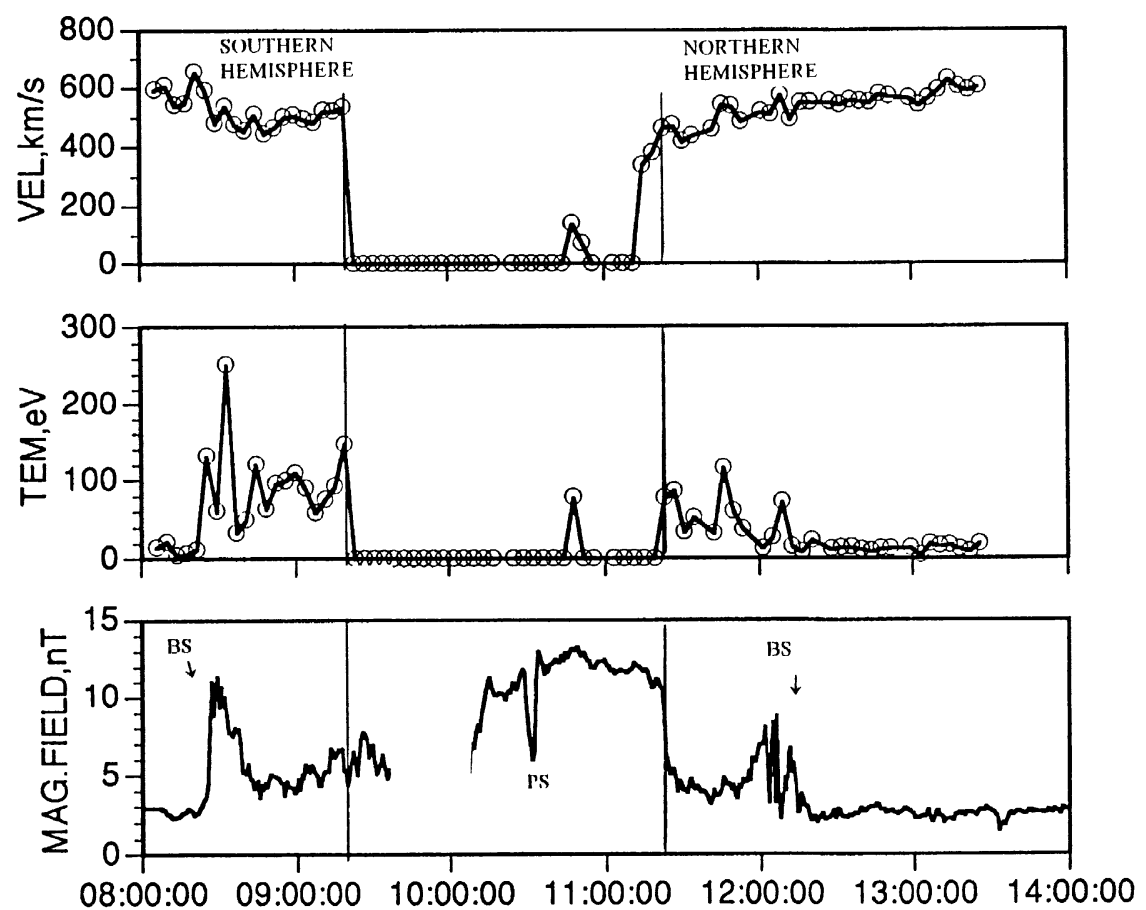

Fig. 11. Example of asymmetrical magnetosheath flow in the northern and southern hemispheres (March 16, 1989).

high magnetic latitudes. It is worth noting that the ASPERA data were available only on ten of 32 polar orbits identified from the magnetic field measurements. Figure 10(a) presents energy-time spectrograms of proton and electron fluxes, the $B_{X}$-component and the magnetic field value in the polar magnetosheath on March 13, 1989. In the inbound leg of the orbit, the spacecraft crossed the BS and the magnetosheath in the southern polar region $\left(\theta=271^{\circ}\right)$. The outbound pass crossed the northern cap $\left(\theta=122^{\circ}\right)$. A small asymmetry $\left(X_{\text {in }}=0.145 R_{\mathrm{M}}, X_{\text {out }}=-0.26 R_{\mathrm{M}}\right)$ of the BS is observed. The spectrogram of the proton fluxes clearly demonstrates a distinct difference in flow in these two regions. Reacceleration of plasma occurs at high southern magnetic latitudes, while, at the northern latitudes, plasma passing the bow shock is slowed down. The solar wind conditions for this period were rather unusual. The number density was very low $\left(n_{\mathrm{sw}} \sim 0.3 \mathrm{~cm}^{-3}\right)$ and the solar wind speed was high $(\sim 890 \mathrm{~km} / \mathrm{s})$. The low value of $\beta$ $\left(\beta_{i}=8 \pi k T_{i} / B^{2} \sim 0.1\right)$ in the solar wind enhances the role of magnetic forces which supply a flow asymmetry (Tanaka, 1993). Figure 10(b) presents normalized values of fluid parameters (the electron number density, $n_{\mathrm{e}}$ and the proton bulk velocity, $V_{\mathrm{p}}$ ) on the inbound leg of the orbit. The BS was crossed at $\sim 16: 10$ UT. Then, the shocked plasma is accelerated and the bulk velocity achieves and, even, exceeds the speed of the undisturbed solar wind. A new transition occurs at 17:15 UT. Signatures of the transition resemble the plasma behavior in the simulation runs across the secondary shock (Fig. 9). A heating of protons and electrons (Fig. 9(a)) indicates to a shock-like transition. However, the plasma velocity drops, seen to be coincide with the sharp increase in the density at the BS, is not observed when the density increases at the "secondary shock". The velocity does not drop until the density drops down. Similar variations of the number density and the bulk velocity are observed in the simulations with lower values of $\beta$ (Fig. 9(b)). The decrease of the magnetic field value also confuses the identification of the discontinuity as a classical shock.

Figure 11 presents another example of the flow asymmetry for low $\beta$-regime. The orbit (March 16, 1989) passed high latitudes $\left(n_{\mathrm{sw}} \sim 0.6 \mathrm{~cm}^{-3}, V_{\mathrm{sw}} \sim 600 \mathrm{~km} / \mathrm{s}\right)$. The inbound and outbound shock/magnetosheah were crossed in the southern and northern hemispheres respectively. The inbound BS was observed at $X_{\text {in }}=-0.02 R_{\mathrm{M}}$ and $\theta=239^{\circ}$. The outbound BS was documented at $X_{\text {out }}=-0.47 R_{\mathrm{M}}$ and $\theta=140^{\circ}$. A distinct asymmetry of the magnetosheath flow with plasma reacceleration in the south hemisphere and a smooth slow down in the north hemisphere is clearly seen.

\section{Summary}

Recently developed 3D hybrid and MHD simulations predict some interesting asymmetry features near Mars. Because of small size of Mars, effects of finite gyroradius of the solar wind protons may become essential. The 3D particle code simulations (Brecht, 1997a, b) have found that kinetic effects provide strong asymmetry of the bow shock with the large flare in the direction opposite to the motional electric field. However, tests made on the base of the Phobos2 data show that the modeling findings are not well reproduced. The location of the BS appears to be often observed in larger distances in the hemisphere, in which the motional electric field is pointed out the equatorial plane. An absence of the pronounced asymmetry with a bulge in the opposite hemisphere, probably, indicates to a contribution of mass- 
loading, which was not implemented to the model. Recent 3D hybrid simulations (Brecht, 1997c) with an addition of the planetary proton population have revealed a decrease of asymmetry associated with kinetic effects. The $2 \mathrm{D}$ bi-ionMHD and hybrid simulations show that sign of asymmetry varies for different regimes of mass-loading. The observations show that many factors control the position of the Martian BS. Their combined effect leads to a strong variability of its location. Besides a north-south asymmetry due to action of the motional electric field, the evident asymmetry between quasiperpendicular and quasiparallel shocks is revealed. Further observations and a 3D-modeling with an inclusion of heavy ion planetary population are necessary for better understanding of action of different controlling factors which interfere the general pattern of the Martian bow shock.

The 3D-MHD model of the solar wind interaction with Mars predicts the asymmetry of the shocked plasma flow in the equatorial and polar magnetosheath. The simulations show the importance of $\boldsymbol{j} \times \boldsymbol{B}$ forces for the plasma inflow into the wake. These forces provide plasma reacceleration in polar region. The measurements made on Phobos- 2 found cases of plasma reacceleration at high latitudes only for low $\beta$ solar wind when effects related with $\boldsymbol{j} \times \boldsymbol{B}$ forces are expected to be stronger. However, the model does not support north/south asymmetry in the magnetosheath flow. It is assumed that mass-loading, which was not implemented to the model, suppresses efficiency of reacceleration and modifies the pattern of flow closure into the wake, as compared with the results by Tanaka (1993). Shocked solar wind effectively loses its momentum in the process of interaction with planetary ions (Lundin et al., 1991; Lundin and Dubinin, 1992; Dubinin et al., 1996a, b). Another interesting feature of the model is a secondary shock formed near the wake. Signatures of a discontinuity-like transition at the border of the proton cavity are observed, although the structure of the transition appears to be more complicated and can not be identified as MHD shock, because of a decrease of the magnetic field value and strong field rotation. Dubinin et al. (1996a) showed that a termination of proton flows across this boundary is accompanied by increase of fluxes of planetary ions. The ion composition transition is often characterized by shock signatures, as increase of the proton temperature (Dubinin et al., 1996a) and enhancement of the plasma wave turbulence (Trotignon et al., 1996), but physics of the transition is unclear yet.

Acknowledgments. The authors wish to express thanks to the ISSI in Bern for the support in the collaboration in the framework of Visiting Science Program. The authors thank S. Livi for the providing of the plasma data from the TAUS experiment which were used for evaluations of the solar wind conditions. The comments of the referees, which helped to improve the paper are also acknowledged.

\section{References}

Alexander, C. J., J. G. Luhmann, and C. T. Russell, Interplanetary field control of the Venus bow shock: Evidence for comet-like pickup, Geophys. Res. Lett., 13, 917-920, 1986.

Bogdanov, A., K. Sauer, K. Baumgärtel, and K. Srivastava, Plasma structures at weakly outgassing comets-results from bi-ion fluid analysis, Planet. Space Sci., 44, 519-528, 1996.

Brecht, S. H., Hybrid simulations of the magnetic topology of Mars, $J$. Geophys. Res., 102, 4743-4750, 1997a.
Brecht, S. H., Solar wind proton deposition into the Martian atmosphere, $J$. Geophys. Res., 102, 11287-11294, 1997b.

Brecht, S. H., The solar wind interaction with Mars: 3-D hybrid, in Abstracts for the 8th Scientific Assembly of IAGA, p. 431, Uppsala, Sweden, 1997c.

Cloutier, P. A., Solar wind interaction with planetary ionospheres, in Solar Wind Interaction with Planets Mercury, Venus, Mars, pp. 111-119, NASA, Spec. Publ. SP-397, 1976.

Delva, M., U. Nischelwitzer, and K. Schwingenschuh, Despinning and tests of the MAGMA data in orbits around Mars, IWF-9407 report of IKI, Graz, Austria, 1994

Dolginov, S. S., E. Dubinin, Ye. Yeroshenko, P. Israelevich, and I. Podgorny, About the configuration of the magnetic field in the Venus tail, Kosmi. Issled., 19, 624-633, 1981.

Dubinin, E., K. Sauer, R. Lundin, O. Norberg, J.-G. Trotignon, K. Schwingenschuh, M. Delva, and W. Riedler, Plasma characteristics of the boundary layer in the martian magnetosphere, J. Geophys. Res., 101, 2706127075, 1996a.

Dubinin, E., K. Sauer, R. Lundin, K. Baumgärtel, and A. Bogdanov, Structuring of the transition region (plasma mantle) of the Martian magnetosphere, Geophys. Res. Lett., 23, 785-788, 1996 b.

Lipatov, A., K. Sauer, and K. Baumgärtel, 2.5 hybrid code simulation of the solar wind interaction with weak comets and related objects, Adv. Space Res., 20, (2)279-(2)282, 1997.

Lundin, R. and E. Dubinin, Phobos-2 results on the ionospheric plasma escape from Mars, Adv. Space Res., 12, (9)255-(9)263, 1992.

Lundin, R., E. Dubinin, H. Koskinen, O. Norberg, N. Pissarenko, and S. Barabash, On the momentum transfer on the solar wind to the Martian topside ionosphere, Geophys. Res. Lett., 18, 1059-1062, 1991.

McComas, D. E., H. E. Spence, C. T. Russell, and M. A. Saunders, The average magnetic field draping and plasma properties of the Venus magnetotail, J. Geophys. Res., 91, 7939-7953, 1986.

Phillips, J. L., J. G. Luhmann, C. T. Russell, and K. R. Moore, Finite Larmor radius effects on ion pickup at Venus, J. Geophys. Res., 92, 9920-9930, 1987.

Phillips, J. L., J. G. Luhmann, W. C. Knudsen, and L. H. Brace, Asymmetries in the location of Venus ionopause, J. Geophys. Res., 93, 3927-3941, 1988 .

Romanov, S., Asymmetry of the region interaction of the solar wind with Venus according to the data of the Venera-9, Venera-10 spacecraft, Kosmi. Issled., 16, 318-319, 1978.

Russell, C. T., E. Chou, J. G. Luhmann, P. Gaziz, L. H. Brace, and W. R. Hoegy, Solar and Interplanetary control of the location of the Venus bow shock, J. Geophys. Res., 93, 5461-5469, 1988.

Saunders, M. A. and C. T. Russell, Average dimensions and magnetic structures of the distant Venus magnetotail, J. Geophys. Res., 91, 5589-5604, 1986.

Schwingenschuh, K., W. Riedler, H. Lichtenegger et al., Martian bow shock: Phobos observations, Geophys. Res. Lett., 17, 889-892, 1990.

Slavin, J. A., D. S. Intriligator, and E. J. Smith, Pioneer-Venus Orbiter magnetic field and plasma observations in the Venus magnetotail, J. Geophys. Res., 94, 2383-2398, 1989.

Slavin, J. A., M. Verigin, K. Gringuuz et al., The solar wind interaction with Mars: The Phobos-2 bow shock observations on 24 March 1989, in Plasma Environments of Non-Magnetic Planets, edited by T. Gombosi, pp. 279-283, COSPAR Colloq. Ser., vol. 4, Pergamon Press, N.Y., 1993.

Tanaka, T., Configurations of the solar wind flow and magnetic field around the planets with no magnetic field: Calculation by a new MHD simulation scheme, J. Geophys. Res., 98, 17251-17262, 1993.

Trotignon, J.-G., E. Dubinin, R. Grard, S. Barabash, and R. Lundin, Martian planetopause as seen by the plasma wave system onboard Phobos-2, J. Geophys. Res., 101, 24965-24977, 1996.

Verigin, M. I., K. I. Gringauz, G. A. Kotova et al., The dependence of the martian magnetopause and bow shock on solar wind ram pressure according to Phobos-2 TAUS ion spectrometer measurements, J. Geophys. Res., 98, 1303-1309, 1993.

Zhang, T. L., K. Schwingenschuh, H. Lichtenegger, W. Riedler, C. T. Russell, and J. G. Luhmann, Interplanetary magnetic field control of the Mars bow shock: Evidence for Venus like interaction, J. Geophys. Res., 96, 11265-11269, 1991.

E. Dubinin (e-mail: dubinin@helene.mpae.gwdg.de), K. Sauer (e-mail: sauer@linax1.mpae.gwdg.de), M. Delva (e-mail: delva@fiwf01.tu-graz. ac.at), and T. Tanaka 